\title{
THE GOLOD PROPERTY FOR STANLEY-REISNER RINGS IN VARYING CHARACTERISTIC
}

\author{
LUKAS KATTHÄN
}

\begin{abstract}
We show that the Golod property of a Stanley-Reisner ring can depend on the characteristic of the base field. More precisely, for every finite set $T$ of prime numbers we construct simplicial complexes $\Delta$ and $\Gamma$, such that $\mathbb{K}[\Delta]$ is Golod exactly in the characteristics in $T$ and $\mathbb{K}[\Gamma]$ is Golod exactly in the characteristics not in $T$.

Along the way, we show that a one-dimensional simplicial complex is Golod if and only if it is chordal.
\end{abstract}

\section{INTRODUCTION}

Let $\mathbb{K}$ be a field and $S=\mathbb{K}\left[X_{1}, \ldots, X_{n}\right]$ be the polynomial ring, endowed with the standard $\mathbb{Z}$-grading. For a homogeneous ideal $\mathfrak{a}$, let $A:=S / \mathfrak{a}$ be the quotient ring. As $A$ is a standard-graded algebra, its Tor-algebra $\operatorname{Tor}_{*}^{A}(\mathbb{K}, \mathbb{K})$ inherits a $\mathbb{Z}$-grading. The Poincaré-series of $A$ is the formal power series

$$
P_{A}(t, x)=\sum_{i, j \geq 0}\left(\operatorname{dim}_{\mathbb{K}} \operatorname{Tor}_{i}^{A}(\mathbb{K}, \mathbb{K})_{j}\right) t^{i} x^{j},
$$

where $\operatorname{Tor}_{i}^{A}(\mathbb{K}, \mathbb{K})_{j}$ denotes the homogeneous component of $\operatorname{Tor}_{i}^{A}(\mathbb{K}, \mathbb{K})$ in degree $j$. The algebra $A$ is called Golod if the following holds:

$$
P_{A}(t, x)=\frac{(1+t x)^{n}}{1-\sum_{i \geq 1} \sum_{j \geq 0} \operatorname{dim}_{\mathbb{K}}\left(\operatorname{Tor}_{i}^{S}(A, \mathbb{K})_{j}\right) t^{i+1} x^{j}}
$$

In general, $P_{A}(t, x)$ is componentwise bounded above by the right-hand side of (1), as it was shown by Serre.

Golod algebras are surprisingly common. For example, it has been proven by Herzog and Huneke [HH13] that if $\mathfrak{a} \subseteq S$ is a homogeneous ideal, then $S / \mathfrak{a}^{k}$ is Golod for every $k>1$. Further, Herzog, Welker and Reiner showed in [HRW99] that $S / \mathfrak{a}$ is Golod if $\mathfrak{a}$ has a componentwise linear resolution. We refer the reader to the survey article [Avr98] by Avramov for more information on Golod algebras.

In [Jö106] and [BJ07] Berglund and Jöllenbeck considered the Golod property for Stanley-Reisner rings. They give a combinatorial characterization of Golodness in the class of flag simplicial complexes, which in particular implies that the Golod property of these complexes does not depend on the field $\mathbb{K}$ of coefficients. Thus it seems natural to ask whether one can find a combinatorial description of the Golod property for Stanley-Reisner rings of general simplicial complexes. The general expectation seems to be that this is not the case, i.e. for sufficiently complicated complexes the Golod property might depend $\mathbb{K}$. However, no example of this phenomenon was known.

2010 Mathematics Subject Classification. Primary: 05E40; Secondary: 13D02,13F55.

Key words and phrases. Golod Ring; Stanley-Reisner ring; Monomial ideal; Characteristic. 
In the present note, we provide a construction for such examples. More precisely, we prove the following:

Theorem 3.4. Let $T$ be a finite set of prime numbers.

(1) There exists a simplicial complex $\Delta$ such that $\mathbb{K}[\Delta]$ is Golod if and only if char $\mathbb{K} \in T$.

(2) Also, there exists a simplicial complex $\Gamma$ such that $\mathbb{K}[\Gamma]$ is Golod if and only if char $\mathbb{K} \notin T$.

We remark that for many properties of $\mathbb{K}[\Delta]$, for example the property of having a componentwise linear resolution or being Cohen-Macaulay, only the second case can happen. Our proof is constructive, but for convenience we also give two explicit examples for the case $T=\{2\}$ in Section 4 .

Let us explain the mechanisms which cause these two cases. By a result of Iriye and Kishimoto [IK14], the Golod property depends on the vanishing of certain maps between homology groups, see Proposition 2.1. On the one hand, it might happen that these homology groups are torsion groups and thus vanish in all but finitely many characteristics. On the other hand, a map between the free parts of the homology groups might be the multiplication by some number $N$. In this case, the map vanishes exactly for the finitely many prime divisors of $N$.

Given our main result, one might be tempted to ask if the finiteness assumption is necessary. In other words, one could ask if there exists a simplicial complex which is Golod in infinitely many characteristics, and non-Golod in infinitely many other characteristics. Our second result gives a negative answer to this question. Thus, the complexes constructed in Theorem 3.4 are "worst possible".

Proposition 3.6. For a simplicial complex $\Delta$, the following holds:

(1) The Golod property of $\mathbb{K}[\Delta]$ depends only on the characteristic of $\mathbb{K}$. More precisely, if $\mathbb{K}$ and $\mathbb{K}^{\prime}$ are two fields with the same characteristic, then $\mathbb{K}[\Delta]$ is Golod if and only if $\mathbb{K}^{\prime}[\Delta]$ is Golod.

(2) If $\mathbb{Q}[\Delta]$ is Golod, then $\mathbb{F}_{p}[\Delta]$ is Golod for all but at most finitely many primes $p$.

(3) If $\mathbb{Q}[\Delta]$ is not Golod, then $\mathbb{F}_{p}[\Delta]$ is Golod for at most finitely many primes $p$.

Here, $\mathbb{F}_{p}$ denotes the field with $p$ elements.

This paper is organized as follows. In Section 2, we collect some background information on Golod rings. In particular, we derive some useful criteria for deciding whether a Stanley-Reisner ring is Golod. In the third section, we first consider the contributions of the one-skeleton of a simplicial complex to the Koszul homology. As a by-product, we show that a one-dimensional simplicial complex is Golod if and only if it is chordal. After that we prove Theorem 3.4 and Proposition 3.6. In Section 4, two explicit examples of Stanley-Reisner rings whose Golodness depends on the field are provided. Finally, in the last section we shortly discuss a relation to decomposition $k$-chordal complexes [ANS15] and pose a question for an improved criterion for Golodness. 


\section{Preliminaries about the Golod property for Stanley-Reisner RINGS}

In this section, we recall some facts about Golod Stanley-Reisner rings. We refer the reader to [Avr86] and [Avr98] for a comprehensive treatment of general Golod rings.

Let $\Delta$ be a fixed simplicial complex. We denote its set of vertices by $V(\Delta)$ and set $n:=\# V(\Delta)$. Let $\mathbb{K}[\Delta]=S / I_{\Delta}$ its Stanley-Reisner ring (over some fixed field $\mathbb{K})$, cf. Chapter 5 of [BH98]. Here, $S=\mathbb{K}\left[X_{v}: v \in V(\Delta)\right]$ is a polynomial ring and

$$
I_{\Delta}=\left(\prod_{v \in M} X_{v}: M \subseteq V(\Delta), M \notin \Delta\right)
$$

is the Stanley-Reisner ideal of $\Delta . \mathbb{K}[\Delta]$ carries a natural $\mathbb{N}^{n}$-grading and we will occasionally identify squarefree multidegrees with subsets of $[n]:=\{1, \ldots, n\}$.

Let further $K_{\mathbb{K}[\Delta]}$ denote the Koszul complex of $\mathbb{K}[\Delta]$, see [BH98, Chapter 1.6]. It carries natural "homological" $\mathbb{N}$-grading, in addition to the "internal" $\mathbb{N}^{n}$ grading inherited from $\mathbb{K}[\Delta]$. For a homogeneous element $a \in K_{\mathbb{K}[\Delta]}$, we denote by $|a|$ its homological degree and by $\operatorname{deg} a$ its internal multidegree. It is wellknown that $K_{\mathbb{K}[\Delta]}$ is an $S$-algebra, which is skew-commutative with respect to the homological grading. Also, the multiplication on $K_{\mathbb{K}[\Delta]}$ induces a multiplication on its homology $H_{*}\left(K_{\mathbb{K}[\Delta]}\right)=\operatorname{Tor}_{*}^{S}(\mathbb{K}[\Delta], \mathbb{K})$.

By definition, $\mathbb{K}[\Delta]$ is called Golod if the equality (1) of power series holds. Golod [Gol62] showed that this is equivalent to the condition that all Massey products on the homology $H_{*}\left(K_{\mathbb{K}[\Delta]}\right)$ are trivial. We will recall the definition of the latter below in the proof of Lemma 2.3. For now, we only point out that the second Massey product of two elements $a_{1}, a_{2} \in H_{*}\left(K_{\mathbb{K}[\Delta]}\right)$ is just the usual product.

Hochster [Hoc77] gave a topological interpretation of the homogeneous strands of the Koszul complex $K_{\mathbb{K}[\Delta]}$. Namely, the strand $\left(K_{\mathbb{K}[\Delta]}\right)_{I}$ in degree $I \subseteq[n]$ is isomorphic to the simplicial cochain complex on the restriction $\left.\Delta\right|_{I}$, where the cohomological grading on the latter is reversed and shifted, such that the vertices of $\left.\Delta\right|_{I}$ sit in degree \#I-1. In particular, passing to homology yields Hochster's formula

$$
H_{i}\left(K_{\mathbb{K}[\Delta]}\right)_{I}=\tilde{H}^{\# I-1-i}\left(\left.\Delta\right|_{I} ; \mathbb{K}\right) .
$$

This equation yields a useful interpretation of the product on $H_{*}\left(K_{\mathbb{K}[\Delta]}\right)$, cf. [BP12, Proposition 3.2.10]. Indeed, the latter is in fact induced from the inclusions $\left.\left.\left.\Delta\right|_{I \cup J} \hookrightarrow \Delta\right|_{I} * \Delta\right|_{J}$ for all $I, J \neq \emptyset$ with $I \cap J=\emptyset$, where $\Delta * \Gamma:=\{F \cup G: F \in$ $\Delta, G \in \Gamma\}$ denotes the join. Since we are working over a field, we may replace cohomology by homology. This leads to the following useful criterion for the vanishing of the product:

Proposition 2.1 (Proposition 6.3, [IK14]). The product on $H_{*}\left(K_{\mathbb{K}[\Delta]}\right)$ is trivial if and only if the inclusion $\left.\left.\left.\Delta\right|_{I \cup J} \hookrightarrow \Delta\right|_{I} * \Delta\right|_{J}$ induces the zero map in homology with coefficients in $\mathbb{K}$ for all $\emptyset \neq I, J \subset V(\Delta)$ with $I \cap J=\emptyset$.

Notation. Let $i \in \mathbb{N}$ and let $I, J$ be two non-empty disjoint subsets of $V(\Delta)$. We write $\varphi_{i}^{I, J}: \tilde{H}_{i}\left(\left.\Delta\right|_{I \cup J} ; \mathbb{K}\right) \rightarrow \tilde{H}_{i}\left(\left.\left.\Delta\right|_{I} * \Delta\right|_{J} ; \mathbb{K}\right)$ for the map induced by the inclusion $\left.\left.\left.\Delta\right|_{I \cup J} \hookrightarrow \Delta\right|_{I} * \Delta\right|_{J}$. 
The following is just a reformulation of Proposition 2.1:

Corollary 2.2. The product on $H_{*}\left(K_{\mathbb{K}[\Delta]}\right)$ is trivial if and only if $\varphi_{i}^{I, J}=0$ for all $i \in \mathbb{N}$ and all nonempty disjoint $I, J \subset V(\Delta)$.

The following lemma allows us to concentrate on the product on $H_{*}\left(K_{\mathbb{K}[\Delta]}\right)$, so we do not need to consider the higher Massey products.

Lemma 2.3. Assume that $\operatorname{dim} \Delta \leq 2$ and that for any two disjoint non-empty sets $I, J \subset V(\Delta)$, at least one of the two complexes $\left.\Delta\right|_{I}$ and $\left.\Delta\right|_{J}$ is connected. Then $\mathbb{K}[\Delta]$ is Golod if and only if the product on $H_{*}\left(K_{\mathbb{K}[\Delta]}\right)$ is trivial.

Proof. The necessity is clear, so we only consider the sufficiency.

Let us recall the definition of the Massey products. As mentioned above, the second Massey product of two elements $a_{1}, a_{2} \in H_{*}\left(K_{\mathbb{K}[\Delta]}\right)$ is just the usual product. Let us denote it by $\mu_{2}\left(a_{1}, a_{2}\right)$. For $n \geq 3$, the $n$-th Massey product is a partially defined set-valued function, which assigns to $n$ elements $a_{1}, \ldots, a_{n} \in$ $H_{*}\left(K_{\mathbb{K}[\Delta]}\right)$ a set $\mu_{n}\left(a_{1}, \ldots, a_{n}\right) \subset H_{*}\left(K_{\mathbb{K}[\Delta]}\right)$. It is defined if there exist elements $a_{i j} \in K_{\mathbb{K}[\Delta]}$ for $1 \leq i \leq j \leq n$, such that $d a_{i i}=0,\left[a_{i i}\right]=a_{i}$ and

$$
d a_{i j}=\sum_{v=i}^{j} \bar{a}_{i v} a_{v j},
$$

where $\bar{a}=(-1)^{|a|+1}$. Then $\sum_{v=1}^{n} \bar{a}_{i v} a_{v j}$ is called a Massey product of $a_{1}, \ldots, a_{n}$ and $\mu_{n}\left(a_{1}, \ldots, a_{n}\right)$ is the set of all these elements. One says that the Massey product is trivial up to $n$, if for all $2 \leq i \leq n$ and all for all $i$-tuples $a_{1}, \ldots, a_{i} \in$ $H_{*}\left(K_{\mathbb{K}[\Delta]}\right)$, the set $\mu_{i}\left(a_{1}, \ldots, a_{i}\right)$ is defined and contains only zero. Finally, we say that $H_{*}\left(K_{\mathbb{K}[\Delta]}\right)$ has trivial Massey products if it is trivial for all $n$. We will use the following properties of the Massey products, which follow from the definition and are well-known:

(a) If the Massey product is trivial up to $n-1$, then $\mu_{n}\left(a_{1}, \ldots, a_{n}\right)$ is defined for all $n$-tuples $a_{1}, \ldots, a_{n}$ and contains only one element. So, in this case one has an actual map $\mu_{n}: H_{*}\left(K_{\mathbb{K}[\Delta]}\right)^{\otimes n} \rightarrow H_{*}\left(K_{\mathbb{K}[\Delta]}\right)$.

(b) If $\mu_{n}\left(a_{1}, \ldots, a_{n}\right)$ is defined, then every element in this set has multidegree $\sum_{i} \operatorname{deg} a_{i}$ and homological degree $\sum_{i}\left(\left|a_{i}\right|+1\right)-2$.

(c) If $\mu_{n}\left(a_{1}, \ldots, a_{n}\right)$ is defined and one of the $a_{i}$ is zero, then $\mu_{n}\left(a_{1}, \ldots, a_{n}\right)$ contains zero.

By induction, assume that the Massey product is trivial up to $n-1$ for some $n \geq 3$, so $\mu_{n}$ is a map by (a). Then (b) implies that $\mu_{n}$ is zero in any nonsquarefree degree. In a squarefree multidegree $I$, Hochster's formula (2) implies that $\mu_{n}$ decomposes into a direct sum of maps of the form

$$
\bigoplus \tilde{H}_{i_{1}, \ldots, i_{n}}\left(\left.\Delta\right|_{I_{1}} ; \mathbb{K}\right) \otimes \cdots \otimes \tilde{H}_{i_{n}}\left(\left.\Delta\right|_{I_{n}} ; \mathbb{K}\right) \rightarrow \tilde{H}_{i}\left(\left.\Delta\right|_{I} ; \mathbb{K}\right)
$$

where $I_{1} \cup I_{2} \cup \cdots \cup I_{n}=I$ is a disjoint decomposition of $I$ with $I_{j} \neq \emptyset$ for all $j$, and the sum runs over all $0 \leq i_{1}, \ldots, i_{n} \leq n$ with $\sum_{j} i_{j}=i-1$. As $\operatorname{dim} \Delta=2$, it follows from (3) and (c) that for $\mu_{n}$ being not trivial, there have to exist non-empty disjoint subsets $I_{1}, \ldots, I_{n} \subset V$, such that either

- $\tilde{H}_{0}\left(\left.\Delta\right|_{I_{i}} ; \mathbb{K}\right) \neq 0$ for all $i$, or

- $\tilde{H}_{0}\left(\left.\Delta\right|_{I_{i}} ; \mathbb{K}\right) \neq 0$ for all $i$ but one, say $i_{1}$, and $\tilde{H}_{1}\left(\left.\Delta\right|_{I_{i_{1}}} ; \mathbb{K}\right) \neq 0$. 
In both cases, there are at least two disjoint non-empty subsets $I, J \subset V$ such that both $\left.\Delta\right|_{I}$ and $\left.\Delta\right|_{J}$ are disconnected, contradicting our assumption.

Remark 2.4. (1) Berglund and Jöllenbeck showed in [BJ07, Theorem 5.1] that in general $\mathbb{K}[\Delta]$ is Golod if and only if the product on $H_{*}\left(K_{\mathbb{K}[\Delta]}\right)$ is trivial, so the assumptions of Lemma 2.3 are superfluous. However, we prefer to prove our main result independently of that result. The reason is that Lemma 2.3 has a very simple proof and is sufficient for our present purpose, while the proof of [BJ07, Theorem 5.1] is rather long and involved.

(2) It is possible (and perhaps preferable) to consider Massey products in the language of $A_{\infty}$-algebras, cf. [LPWZ09, Val14]. This allows one to replace the classical Massey products with maps $\mu_{n}: H_{*}\left(K_{\mathbb{K}[\Delta]}\right)^{\otimes n} \rightarrow H_{*}\left(K_{\mathbb{K}[\Delta]}\right)$ which are always defined and no longer set-valued. However, for the purpose of the present paper the classical approach to Massey products is sufficient.

\section{Proof of the MAin Results}

In this section, we first give some useful results concerning the maps $\varphi_{i}^{I, J}$. After these preparations, our main result Theorem 3.4 is be proven. In the last part of the section, we prove the above mentioned Proposition 3.6

We start with some notation. For a simplicial complex $\Delta$, we write $C_{i}(\Delta ; \mathbb{K})$ for the vector space of simplicial $i$-chains of $\Delta$. We say that an $i$-cycle $c \in C_{i}(\Delta, \mathbb{K})$ contains an $i$-face $F$ if it has a non-zero coefficient in $c$. An $i$-cycle $c \in C_{i}(\Delta, \mathbb{K})$ is called complete [ANS15] if it is the boundary of a $(i+1)$-simplex (which does not need to be a face of $\Delta$ ). The following is a simple criterion to decide the vanishing or non-vanishing of $\varphi_{i}^{I, J}$ in some cases.

Lemma 3.1. Let $\Delta$ be a simplicial complex, let $\emptyset \neq I, J \subseteq V(\Delta)$ with $I \cap J=\emptyset$ and let $c \in C_{i}\left(\left.\Delta\right|_{I \cup J} ; \mathbb{K}\right)$ be an $i$-cycle, for some $i$.

(1) If $c$ is complete, then $\varphi_{i}^{I, J}(c)=0$.

(2) If c contains an $i$-face $F$, such that $F \cap I$ and $F \cap J$ are both facets of $\left.\Delta\right|_{I}$ and $\left.\Delta\right|_{J}$, respectively, then $\varphi_{i}^{I, J}(c) \neq 0$.

Proof. (1) First, assume that some vertices of $c$ are in $I$ and some are in $J$, i.e., there are non-empty faces $\left.\sigma \in \Delta\right|_{I},\left.\tau \in \Delta\right|_{J}$ such that the support of $c$ equals $\sigma \cup \tau$. It holds that $\varphi_{i}^{I, J}(c)=\partial(\sigma \cup \tau)$ because $c$ is complete. Hence $\varphi_{i}^{I, J}(c)$ is a boundary.

Next, consider the case that all vertices of $c$ are in one of the sets, say $I$. Then $c$ is the boundary of the cone $c *\{w\}$ for any vertex $w \in J$.

(2) Under the given hypothesis $F$ is a facet of $\left.\left.\Delta\right|_{I} * \Delta\right|_{J}$. Hence $\varphi_{i}^{I, J}$ (c) is not a boundary.

It was proven in [BJ07, Proposition 6.4] that the 1-skeleton of a Golod simplicial complex is chordal. The following extends this result.

Proposition 3.2. For a simplicial complex $\Delta$, the following are equivalent:

(1) The 1-skeleton of $\Delta$ is a chordal graph.

(2) $\varphi_{1}^{I, J}=0$ for all non-empty disjoint subsets $I, J \subset V(\Delta)$. 


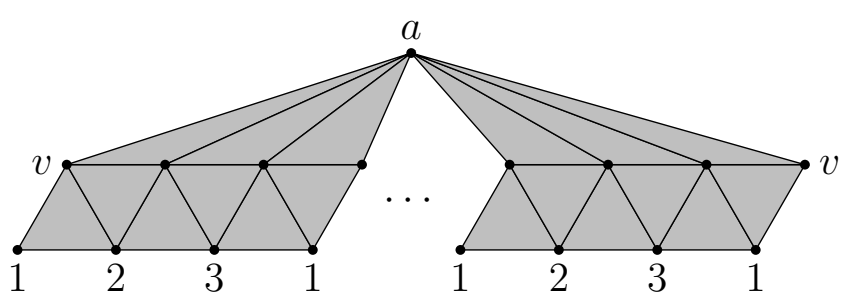

Figure 1. The complex $\Delta_{1}$ used in the proof of Theorem 3.4. The sequence $1,2,3$ repeats $N$ times.

Proof. $\quad(1) \Rightarrow(2)$ If the 1-skeleton of $\Delta$ is a chordal graph, then every 1-cycle can be written as a sum of "triangles". But a triangle is a complete 1-cycle, so the claim follows form Lemma 3.1.

$(2) \Rightarrow(1)$ Assume that the 1 -skeleton of $\Delta$ contains a chordless cycle $c$ of length at least 4 . Let $v$ be any vertex of $c$ and let $w_{1}, w_{2}$ denote its two neighbors. Set $I:=\left\{w_{1}, w_{2}\right\}$ and let $J$ be the set of all other vertices of $c$. Note that the vertices $v$ and $w_{1}$ are isolated in $\left.\Delta\right|_{I}$ and $\left.\Delta\right|_{J}$, respectively, because $c$ is chordless.

But the homology class $\tilde{c} \in \tilde{H}_{1}\left(\left.\Delta\right|_{I \cup J} ; \mathbb{K}\right)$ corresponding to $c$ contains the edge $\left\{v, w_{1}\right\}$, hence $\varphi_{1}^{I, J}(\tilde{c}) \neq 0$ by Lemma 3.1 .

It is known that a flag simplicial complex is Golod if and only if its one-skeleton is chordal, see [BJ07, Theorem 6.7]. A one-dimensional simplicial complex does not need to be flag, so the following can be seen as a partial extension of that result.

Corollary 3.3. Let $\Delta$ be a one-dimensional simplicial complex. Then $\mathbb{K}[\Delta]$ is Golod if and only if $\Delta$ is chordal.

After the preparation of the present article I learned that Iriye and Kishimoto proved a stronger version of this corollary in [IK14, Theorem 11.8].

Proof. This is immediate from the foregoing Proposition 3.2 and [BJ07, Theorem 5.1]. Alternatively, if $\Delta$ is chordal, then its Stanley-Reisner ideal is componentwise linear (the degree 2 part is linear by [Frö90] and the regularity is at most $3)$, so $\mathbb{K}[\Delta]$ is Golod, cf. [HRW99].

Now we turn to the proof of our main result:

Theorem 3.4. Let $T$ be a finite set of prime numbers.

(1) There exists a simplicial complex $\Delta$ such that $\mathbb{K}[\Delta]$ is Golod if and only if char $\mathbb{K} \in T$.

(2) Also, there exists a simplicial complex $\Gamma$ such that $\mathbb{K}[\Gamma]$ is Golod if and only if char $\mathbb{K} \notin T$.

Proof. Let $N$ be the product of the elements of $T$.

Let $\Delta_{1}$ be the simplicial complex indicated in Fig. 1, where vertices with the same label are to be identified. Here, the sequence 1,2,3 of the bottom vertices is repeated $N$ times. So, topologically $\Delta_{1}$ is obtained by gluing the boundary of a 2-cell $N$ times around the cycle $\gamma$ formed by the vertices 1,2 and 3 . 
(1) Let $\Delta_{2}$ be the complex obtained by gluing two copies of $\Delta_{1}$ along $\gamma$. We denote the vertex " $a$ " in the second copy of $\Delta_{1}$ by $b$. Further, let $\Delta$ be the complex which is obtained from $\Delta_{2}$ by adding all edges between any two vertices except $a$ and $b$.

Note that $\Delta$ satisfies the hypotheses of Lemma 2.3 , so it is sufficient to show that the product on $H_{*}\left(K_{\mathbb{K}[\Delta]}\right)$ is trivial if and only if char $\mathbb{K}$ divides $N$. We will use Corollary 2.2 for this. For dimension reasons, $\varphi_{i}^{I, J}=0$ for all $i \geq 3$ and all $I, J$. Further, the 1-skeleton of $\Delta$ is complete except for the missing edge from $a$ to $b$, so it is chordal. Hence $\varphi_{1}^{I, J}=0$ for any $I$ and $J$ by Proposition 3.2. To finish the proof, we are going to show that

(a) for $I:=\{a, b\}$ and $J:=V(\Delta) \backslash I$, it holds that $\varphi_{2}^{I, J}$ is zero if and only if char $\mathbb{K} \in T$, and

(b) further, $\varphi_{2}^{I^{\prime}, J^{\prime}}=0$ independently of the field for any two disjoint vertex sets $I^{\prime}, J^{\prime}$ with $\left\{I^{\prime}, J^{\prime}\right\} \neq\{I, J\}$.

We start by showing the first item, so let $I=\{a, b\}$ and let $J=V(\Delta) \backslash I$. Let $\sigma_{1}, \sigma_{2} \in C_{2}(\Delta ; \mathbb{K})$ be the sums of all triangles in the first and the second copy of $\Delta_{1}$, respectively, endowed with suitable signs. Clearly, the boundary of both $\sigma_{1}$ and $\sigma_{2}$ is $N$ times $\gamma$. So $\sigma_{1}$ and $\sigma_{2}$ are both cycles if char $\mathbb{K}$ divides $N$. Further, their difference $\sigma:=\sigma_{1}-\sigma_{2}$ is a cycle, independently of the field.

If char $\mathbb{K} \in T$, then $\tilde{H}_{2}(\Delta ; \mathbb{K})$ is generated by (the classes of) $\sigma_{1}$ and $\sigma_{2}$. The image of $\sigma_{1}$ (resp. $\sigma_{2}$ ) in $\left.\left.\Delta\right|_{I} * \Delta\right|_{J}$ is supported on the contractible subcomplex $\left.\{a\} * \Delta\right|_{J}$ (respectively $\left.\{b\} * \Delta\right|_{J}$ ), so $\varphi_{2}^{I, J}$ sends it to zero. Thus, if char $\mathbb{K} \in T$ then $\varphi_{2}^{I, J}$ is the zero map for the given choice of $I$ and $J$.

Now assume that char $\mathbb{K} \notin T$. Note that $\tilde{H}_{2}(\Delta ; \mathbb{K})=\tilde{H}_{2}\left(\Delta_{2} ; \mathbb{K}\right)$, because the two complexes differ only in their 1-skeleton. So the map $\varphi_{2}^{I, J}$ can be factored as

$$
\tilde{H}_{2}(\Delta ; \mathbb{K}) \rightarrow \tilde{H}_{2}\left(\Delta_{2} ; \mathbb{K}\right) \stackrel{\psi}{\rightarrow} \tilde{H}_{2}\left(\left.\left.\Delta_{2}\right|_{I} * \Delta_{2}\right|_{J} ; \mathbb{K}\right) \rightarrow \tilde{H}_{2}\left(\left.\left.\Delta\right|_{I} * \Delta\right|_{J} ; \mathbb{K}\right) .
$$

The first map is a isomorphism and the last map is injective, because only 1- and 2-cells are added, so no 2-cycle can become a boundary. Hence $\varphi_{2}^{I, J}$ is nonzero if and only if the middle map $\psi$ is nonzero.

Note that $\left.\Delta_{2}\right|_{J}$ retracts onto $\gamma$, hence $\left.\left.\Delta_{2}\right|_{I} * \Delta_{2}\right|_{J}$ retracts onto $\left.\Delta_{2}\right|_{I} * \gamma$, which is just a suspension of $\gamma$, i.e., a 2-sphere. This implies that a generator $\tau$ for $\tilde{H}_{2}\left(\left.\left.\Delta_{2}\right|_{I} * \Delta_{2}\right|_{J} ; \mathbb{K}\right)$ is given by a signed sum of all 2-faces in $\left.\left.\Delta_{2}\right|_{I} * \Delta_{2}\right|_{J}$ containing one of the edges of $\gamma$ and either $a$ or $b$. On the other hand, $\tilde{H}_{2}(\Delta ; \mathbb{K})$ is generated by (the class of) $\sigma:=\sigma_{1}-\sigma_{2}$. By construction, it winds $N$ times around $\gamma$. Thus, under the above mentioned retraction it will be mapped to $\pm N$ times the generator of $\tilde{H}_{2}\left(\left.\Delta_{2}\right|_{I} * \gamma ; \mathbb{K}\right)$ and hence $\psi(\sigma)= \pm N \cdot \tau \neq 0$. In conclusion, $\varphi_{2}^{I, J}$ is zero if and only if char $\mathbb{K} \in T$.

Finally, we show that $\varphi_{2}^{I^{\prime}, J^{\prime}}=0$ for any two disjoint vertex sets $I^{\prime}$, $J^{\prime}$ with $\left\{I^{\prime}, J^{\prime}\right\} \neq\{I, J\}$. By the Künneth formula, $\tilde{H}_{2}\left(\left.\left.\Delta\right|_{I^{\prime}} * \Delta\right|_{J^{\prime}} ; \mathbb{K}\right)$ can be nontrivial only if either $\tilde{H}_{0}\left(\left.\Delta\right|_{I^{\prime}} ; \mathbb{K}\right) \neq 0$ or $\tilde{H}_{0}\left(\left.\Delta\right|_{J^{\prime}} ; \mathbb{K}\right) \neq 0$. Hence we only need to consider the case $I^{\prime}=I=\{a, b\}$.

It remains to show that $\varphi_{2}^{I, J^{\prime}}=0$ if $J^{\prime} \subsetneq V(\Delta) \backslash\{a, b\}$. Let $\omega \in C_{2}\left(\left.\Delta\right|_{I \cup J^{\prime}} ; \mathbb{K}\right)$ be a cycle. If $\omega$ contains a 2 -face $F$, then for every edge of $F$ it also contains another 2 -face with that edge. Using the definition of $\Delta$, it follows that for each copy of $\Delta_{1}$ inside $\Delta, \omega$ contains either all or none of its 2-faces. As $I \cup J^{\prime} \neq V(\Delta), \omega$ 
cannot contain all 2-faces of $\Delta$, so all triangles in $\omega$ lie in the same copy of $\Delta_{1}$, say in the one with vertex $a$. But this implies that the image of $\omega$ in $C_{2}\left(\left.\left.\Delta\right|_{I} * \Delta\right|_{J^{\prime}} ; \mathbb{K}\right)$ it is supported on the contractible subcomplex $\left.\{a\} * \Delta\right|_{J^{\prime}}$, so as above $\varphi_{2}^{I, J^{\prime}}$ sends it to zero.

(2) We proceed similar as in the proof of part (1). $\Delta_{1}$ is still the simplicial complex indicated in Fig. 1 . Let $\Gamma$ be the complex obtained from $\Delta_{1}$ by adding all edges which do not contain $a$.

As above, $\Gamma$ satisfies the hypotheses of Lemma 2.3 and its 1-skeleton is chordal, so we only need to consider the maps $\varphi_{2}^{I, J}$. Let $J \subset V(\Gamma)$ be the set of neighbors of $a$ and let $I:=V(\Gamma) \backslash J$. Here, a neighbor of $a$ is any vertex sharing an edge with it. This time, we are going to show the following:

(a) The map $\varphi_{2}^{I, J}$ is nonzero if and only if char $\mathbb{K} \in T$.

(b) Further, $\varphi_{2}^{I^{\prime}, J^{\prime}}=0$ if char $\mathbb{K} \notin T$ for any two disjoint vertex sets $I^{\prime}, J^{\prime}$ with $\left\{I^{\prime}, J^{\prime}\right\} \neq\{I, J\}$.

We show both items simultaneously. Our description of $\Delta_{1}$ given above shows that

$$
\tilde{H}_{2}(\Gamma ; \mathbb{K})=\tilde{H}_{2}\left(\Delta_{1} ; \mathbb{K}\right)= \begin{cases}\mathbb{K} & \text { if char } \mathbb{K} \in T \\ 0 & \text { otherwise }\end{cases}
$$

So clearly $\varphi_{2}^{I^{\prime}, J^{\prime}}=0$ if char $\mathbb{K} \notin T$ for any two disjoint vertex sets $I^{\prime}, J^{\prime}$ with $I^{\prime} \cup J^{\prime}=V(\Gamma)$, in particular for $I^{\prime}=I$ and $J^{\prime}=J$. Also, for two disjoint vertex sets $I^{\prime}, J^{\prime}$ with $I^{\prime} \cup J^{\prime} \subsetneq V(\Gamma)$, one shows similarly to the argument in the proof of part $(1)$ that $\tilde{H}_{2}\left(\left.\Gamma\right|_{I^{\prime} \cup J^{\prime}} ; \mathbb{K}\right)=0$, so the corresponding map vanishes.

It remains to show that $\varphi_{2}^{I, J} \neq 0$ if char $\mathbb{K} \in T$. In this case, a generator of $\tilde{H}_{2}(\Gamma ; \mathbb{K})$ is given by the sum $\sigma_{1} \in C_{2}(\Gamma ; \mathbb{K})$ of all 2 -faces of $\Gamma$, endowed suitable signs. Now, $\sigma_{1}$ contains a 2-face $F$ which contains $a$. Further, $F \cap I=\left.\{a\} \in \Gamma\right|_{I}$ is isolated and $\left.F \cap J \in \Gamma\right|_{J}$ is a facet, because $\left.\Gamma\right|_{J}$ is one-dimensional. Hence $\varphi_{2}^{I, J}\left(\sigma_{1}\right) \neq 0$ by Lemma 3.1

Example 3.5. Let us illustrate the construction of part (1) in the case $T=\{2\}$. In this case, $\Delta_{1}$ is a real projective plane. One can think of it as being obtained by gluing the star of $a$, which is a disc, on the boundary of a Möbius strip, which is the lower part of $\Delta_{1}$ in Figure 1. Now $\Delta_{2}$ is obtained by gluing two copies of this projective planes along $\gamma$. In this situation $\sigma_{1}$ and $\sigma_{2}$ are the fundamental classes (over $\mathbb{Z} / 2$ ) of the two projective planes.

The map $\left.\left.\left.\Delta_{2} \rightarrow \Delta_{2}\right|_{I} * \Delta_{2}\right|_{J} \rightarrow \Delta_{2}\right|_{I} * \gamma \approx S^{2}$ corresponds to retracting the Möbius strips to $\gamma$. Under this map $\sigma_{1}$ and $\sigma_{2}$ get deformed to "two times" the lower resp. upper hemisphere of the resulting sphere. Hence $\sigma_{1}-\sigma_{2}$ is mapped to two times the fundamental class $\tau$ of $S^{2}$.

It seems a natural question if the finiteness of the set $T$ in Theorem 3.4 is really necessary. In other words, could there be a simplicial complex $\Delta$ such that $\mathbb{K}[\Delta]$ is Golod in infinitely many characteristics and non-Golod in infinitely many other characteristics? Indeed, such a phenomenon is excluded by the following result. For completeness, we also show that the Golod property only depends on the characteristic.

Proposition 3.6. For a simplicial complex $\Delta$, the following holds: 
(1) The Golod property of $\mathbb{K}[\Delta]$ depends only on the characteristic of $\mathbb{K}$. More precisely, if $\mathbb{K}$ and $\mathbb{K}^{\prime}$ are two fields with the same characteristic, then $\mathbb{K}[\Delta]$ is Golod if and only if $\mathbb{K}^{\prime}[\Delta]$ is Golod.

(2) If $\mathbb{Q}[\Delta]$ is Golod, then $\mathbb{F}_{p}[\Delta]$ is Golod for all but at most finitely many primes $p$.

(3) If $\mathbb{Q}[\Delta]$ is not Golod, then $\mathbb{F}_{p}[\Delta]$ is Golod for at most finitely many primes $p$.

Here, $\mathbb{F}_{p}$ denotes the field with $p$ elements.

Proof. The claim follows almost immediately from a characterization of the Golod property given by Berglund in [Ber06, Theorem 3]. We briefly recall this characterization. Let $I:=I_{\Delta}$ be the Stanley-Reisner ideal of $\Delta$ and let $M$ be a minimal set of monomial generators of it. Note that $M$ only depends on $\Delta$ and not on the field $\mathbb{K}$. Recall that the lcm-lattice of $I, L_{I}$, is the lattice of all least common multiples of subsets of $M$, ordered by divisibility. For any monomial $m \in L_{I}$, let $M(\leq m)$ denote the set of those monomials in $M$ which divide $m$. Moreover, for any finite set $N$ of monomials, let $m_{N}$ denote the least common multiple of all elements in $N$. In [Ber06], Berglund associates to each finite set $N$ of monomials a natural number $c(N)$ and a lattice of certain subsets of $N$ which is denoted by $K(N)$. The exact definitions are not relevant here; we only need that these constructions do not depend on the underlying field $\mathbb{K}$. Finally, for a finite poset $P$ we set

$$
\tilde{H}(P ; \mathbb{K})(z):=\sum_{i \geq-1} \operatorname{dim}_{\mathbb{K}}\left(\tilde{H}_{i}(P ; \mathbb{K})\right) z^{i},
$$

where $\tilde{H}^{i}(P ; \mathbb{K})$ is the homology of the order complex of $P$. Theorem 3 of [Ber06] asserts that $\mathbb{K}[\Delta]$ is Golod if and only if for each $m \in L_{I}$, it holds that

$$
\tilde{H}\left((\hat{0}, m)_{L_{I}} ; \mathbb{K}\right)(z)=\sum_{\substack{S \in K(M(\leq m)) \\ m_{S}=m}}(-z)^{c(S)-1} \tilde{H}\left((\hat{0}, S)_{K(M(\leq m))} ; \mathbb{K}\right)(z),
$$

where $(\hat{0}, m)_{L_{I}}$ denotes the lower interval defined by $m$ in $L_{I}$, and similarly $(\hat{0}, S)_{K(M(\leq m))}$ is the lower interval defined by $S$ in $K(M(\leq m))$.

Now we observe that both sides of (4) depend on $\mathbb{K}$ only via the homology of some simplicial complexes. But for any simplicial complex $\Gamma, \tilde{H}_{i}(\Gamma ; \mathbb{K})$ depends only on the characteristic of $\mathbb{K}$. Moreover, it holds that

$$
\operatorname{dim}_{\mathbb{F}_{p}} \tilde{H}_{i}\left(\Gamma ; \mathbb{F}_{p}\right)=\operatorname{dim}_{\mathbb{Q}} \tilde{H}_{i}(\Gamma ; \mathbb{Q})
$$

for all but finitely many primes $p$. Thus, the set of characteristics in which (4) holds is either finite or it has a finite complement, and these two cases can be distinguished by considering it over $\mathbb{Q}$.

\section{TWO EXPLICIT EXAMPLES}

We give two explicit examples of simplicial complexes which show the phenomena observed in the last section. In particular, we evaluate the two sides of (1) in these two examples. For this, we define

$$
f_{\mathrm{RH}}:=\sum_{i \geq 1} \sum_{j \geq 0} \operatorname{dim}_{\mathbb{K}}\left(\operatorname{Tor}_{i}^{S}(A, \mathbb{K})_{j}\right) t^{i} x^{j},
$$


so the right-hand side of (1) is given by

$$
\frac{(1+t x)^{n}}{1-t f_{\mathrm{RH}}}
$$

The polynomial $f_{\mathrm{RH}}$ can easily be determined from the graded Betti numbers of $A$, which we computed in our examples with Macaulay2 [GS]. For the left-hand side of (1), it follows from Theorem 1 of [Ber06] (see also [Bac82]) that it is also of the form

$$
\frac{(1+t x)^{n}}{1-t f_{\mathrm{LH}}}
$$

with some polynomial $f_{\mathrm{LH}}$ in $t$ and $x$. To compute it in our examples, we evaluated the formula (9) of [Ber06] using a computer.

Example 4.1. Let $\Delta$ be the simplicial complex with the facets

$\begin{array}{lllll}124 & 235 & 341 & 452 & 513 \\ 12 a & 23 a & 34 a & 45 a & 51 a \\ 12 b & 23 b & 34 b & 45 b & 51 b\end{array}$

on the vertex set $V=\{1,2,3,4,5, a, b\}$. The generators of the corresponding Stanley-Reisner ideal are

$$
\begin{array}{ccccc}
x_{1} x_{2} x_{3} & x_{2} x_{3} x_{4} & x_{3} x_{4} x_{5} & x_{4} x_{5} x_{1} & x_{5} x_{1} x_{2} \\
x_{1} x_{3} x_{a} & x_{1} x_{4} x_{a} & x_{2} x_{4} x_{a} & x_{2} x_{5} x_{a} & x_{5} x_{3} x_{a} \\
x_{1} x_{3} x_{b} & x_{1} x_{4} x_{b} & x_{2} x_{4} x_{b} & x_{2} x_{5} x_{b} & x_{5} x_{3} x_{b} \\
x_{a} x_{b} & & & &
\end{array}
$$

Geometrically, $\Delta$ is a Möbius strip with two 2-balls glued along its boundary. Using a similar argument as in the proof of part (1) of Theorem 3.4, one shows that $\mathbb{K}[\Delta]$ is Golod if and only if char $\mathbb{K}=2$, where the "critical" sets are $I=\{a, b\}$ and $J=\{1, \ldots, 5\}$. Note that the 1-skeleton of $\Delta$ is already chordal, so we do not need to add additional edges as in the proof of Theorem 3.4. For $\mathbb{K}=\mathbb{Q}$, we computed that

$$
\begin{aligned}
& f_{\mathrm{RH}}=\left(x^{2}+15 x^{3}\right) t+35 x^{4} t^{2}+26 x^{5} t^{3}+\left(5 x^{6}+x^{7}\right) t^{4}, \\
& f_{\mathrm{LH}}=\left(x^{2}+15 x^{3}\right) t+35 x^{4} t^{2}+26 x^{5} t^{3}+5 x^{6} t^{4}-x^{7} t^{5} .
\end{aligned}
$$

As expected, there is a strict inequality in (1). For $\mathbb{K}=\mathbb{F}_{2}$, the ring is Golod, so the equality in (1) is attained. In this case, it holds that

$$
f_{\mathrm{RH}}=f_{\mathrm{LH}}=\left(x^{2}+15 x^{3}\right) t+35 x^{4} t^{2}+\left(26 x^{5}+2 x^{6}\right) t^{3}+\left(7 x^{6}+2 x^{7}\right) t^{4}+x^{7} t^{5}
$$

Note that in this example both sides of (1) depend in the field.

Example 4.2. Let $\Delta$ be the simplicial complex with the facets

$$
\begin{array}{lllll}
124 & 235 & 341 & 452 & 513 \\
12 a & 23 a & 34 a & 45 a & \\
51 b & 1 a b & a 5 b & &
\end{array}
$$

on the vertex set $V=\{1,2,3,4,5, a, b\}$. The generators of the corresponding Stanley-Reisner ideal are

$$
\begin{array}{ccccc}
x_{1} x_{2} x_{3} & x_{2} x_{3} x_{4} & x_{3} x_{4} x_{5} & x_{4} x_{5} x_{1} & x_{5} x_{1} x_{2} \\
x_{1} x_{3} x_{a} & x_{1} x_{4} x_{a} & x_{2} x_{4} x_{a} & x_{2} x_{5} x_{a} & x_{5} x_{3} x_{a} \\
x_{5} x_{1} x_{a} & x_{2} x_{b} & x_{3} x_{b} & x_{4} x_{b} &
\end{array}
$$


This is a triangulation of the real projective plane, which is obtained from the usual 6-vertex triangulation by subdividing the 2-cell $51 a$. It can be shown similar as in the proof of part (2) of Theorem 3.4 that $\mathbb{K}[\Delta]$ is Golod if and only if char $\mathbb{K} \neq 2$. Here, the "critical" sets are $I=\{2,3,4, b\}$ and $J=\{5,1, a\}$.

Again, we provide some numerical data for completeness. For $\mathbb{K}=\mathbb{Q}$, the ring is Golod and it holds that

$$
f_{\mathrm{RH}}=f_{\mathrm{LH}}=\left(3 x^{2}+11 x^{3}\right) t+\left(3 x^{3}+28 x^{4}\right) t^{2}+\left(x^{4}+24 x^{5}\right) t^{3}+7 x^{6} t^{4} .
$$

For $\mathbb{K}=\mathbb{F}_{2}$, it holds that

$$
f_{\mathrm{RH}}=\left(3 x^{2}+11 x^{3}\right) t+\left(3 x^{3}+28 x^{4}\right) t^{2}+\left(x^{4}+24 x^{5}\right) t^{3}+\left(7 x^{6}+x^{7}\right) t^{4}+x^{7} t^{5},
$$

while $f_{\mathrm{LH}}$ is the same as over $\mathbb{Q}$. So in this example, somewhat surprisingly, only one side of (1) depends on the field.

Apart from the equation (1), the triviality of the Massey products on $H_{*}\left(K_{\mathbb{K}[\Delta]}\right)$ and [Ber06, Theorem 3], there is a further well-known characterizations of Golod rings. Namely, a ring $A$ is Golod if and only if its homotopy Lie algebra $\pi^{\geq 2}(A)$ is a free (graded) Lie algebra, cf. Chapter 10 of [Avr98]. It might be instructive to study the structure of the homotopy Lie algebra in these examples.

\section{Concluding Remarks}

5.1. Skeleta and higher chordality. In this section, we give two consequences of Lemma 3.1 which we consider to be of independent interest. In the sequel, $\Delta$ is always a simplicial complex. The first one is the following corollary.

Corollary 5.1. The map $\varphi_{i}^{I, J}$ depends only on the $i$-skeleton of $\Delta$.

Proof. It is clear that the map depends only on the $(i+1)$-skeleton of $\Delta$. Adding $(i+1)$-dimensional simplices to $\Delta$ only turns complete $i$-cycles into boundaries. By Lemma 3.1, all complete $i$-cycles lie in the kernel of $\varphi_{i}^{I, J}$, so this does not affect the map.

Remark 5.2. Based on the preceding corollary, one might be tempted to conjecture that the map $\varphi_{i}^{I, J}$ depends only in the pure $i$-skeleton of $\Delta$. But this is false by the following counterexample.

Consider the join of an empty triangle with an $S^{0}$. This complex is not Golod, as it is a join (or Gorenstein*). But the complex $\Delta$ obtained by adding an edge between the two "poles" is Golod: If the two poles are $I$ and the triangle is $J$, then $\left.\Delta\right|_{I}$ is contractible, so $\left.\left.\Delta\right|_{I} * \Delta\right|_{J}$ is as contractible as well. All proper restrictions of $\Delta$ have no second homology, and the 1-skeleton is chordal. Hence $\Delta$ is Golod.

Adiprasito, Nevo and Samper define in [ANS15] several high-dimensional extensions of the notion of a chordal graph. In particular, they define a simplicial complex to be decomposition $k$-chordal, if every $k$-cycle $z$ can be written as a sum of complete $k$-cycles $\left(z_{i}\right)$, such the vertices of each $\left(z_{i}\right)$ are also vertices of $z$. The sufficiency of Proposition 3.2 extends to this setting:

Proposition 5.3. If $\Delta$ is a decomposition $k$-chordal simplicial complex, then $\varphi_{k}^{I, J}=0$ for all non-empty disjoint subsets $I, J \subset V$. In particular, if $\Delta$ is decomposition $k$-chordal for all $k$, then the product on $H_{*}\left(K_{\mathbb{K}[\Delta]}\right)$ is trivial. 
Proof. This is immediate from Lemma 3.1.

5.2. Degree bounds. By Corollary 2.2, $\mathbb{K}[\Delta]$ is Golod if the maps $\varphi_{i}^{I, J}$ vanish for all $i \in \mathbb{N}$. It is clear that one only has to consider $i \leq \operatorname{dim} \Delta$. Moreover, it is in fact sufficient to consider $i \leq \operatorname{reg} \mathbb{K}[\Delta]-1$, where reg $\mathbb{K}[\Delta]$ denotes the Castelnuovo-Mumford regularity. This is immediate from Hochster's formula, which implies that

$$
\operatorname{reg} \mathbb{K}[\Delta]=\max \left\{j: \tilde{H}_{j-1}\left(\left.\Delta\right|_{I} ; \mathbb{K}\right) \neq 0 \text { for some } I \subseteq V\right\} .
$$

It follows from [BJ07, Theorem 6.5] that if $\Delta$ is flag, then the vanishing of $\varphi_{1}^{I, J}$ (for all $I, J$ ) is sufficient for $\mathbb{K}[\Delta]$ to be Golod. An optimistic generalization of this would be the following assertion: If $\Delta$ has no minimal non-faces of dimension $\geq k$ and $\varphi_{i}^{I, J}=0$ for all $i \leq k$, then $\mathbb{K}[\Delta]$ is Golod. However, this can easily seen to be false. An easy example is the join of two boundaries of $(k-1)$-simplices. Instead we ask we following question, which is motivated by an analogous result in $[\mathrm{ANS15}]$ :

Question 5.4. Let $\Delta$ be a simplicial complex with vertex set $V$. Assume that $\Delta$ has no minimal non-faces of dimension $\geq k$, and assume further that $\varphi_{i}^{I, J}=0$ for all $i \leq 2 k-1$ and all non-empty disjoint subsets $I, J \subseteq V$. Is $\mathbb{K}[\Delta]$ Golod?

\section{ACKNOWLEDGMENT}

The author thanks Sean Tilson for many inspiring discussions and Volkmar Welker for suggesting the topic of the present note. Moreover, the author wishes to thank Yi-Huang Shen and the anonymous referee for several helpful comments.

\section{REFERENCES}

[ANS15] K. A. Adiprasito, E. Nevo, and J. A. Samper. Higher chordality I. From graphs to complexes. arXiv:1503.05620, 2015.

[Avr86] L. L. Avramov. Golod homomorphisms. In Algebra, Algebraic Topology and their Interactions, pages 59-78. Springer, 1986.

[Avr98] L. L. Avramov. Infinite free resolutions. In Six lectures on commutative algebra, pages 1-118. Springer, 1998.

[Bac82] J. Backelin. Les anneaux locaux á relations monomiales ont des séries de PoincaréBetti rationnelles. C.R. Acad. Sci. Paris, 295(11):607-610, 1982.

[Ber06] A. Berglund. Poincaré series of monomial rings. Journal of Algebra, 295(1):211 230, 2006.

[BH98] W. Bruns and J. Herzog. Cohen-Macaulay rings. Cambridge University Press, 1998.

[BJ07] A. Berglund and M. Jöllenbeck. On the Golod property of Stanley-Reisner rings. Journal of Algebra, 315(1):249-273, 2007.

[BP12] V. Buchstaber and T. Panov. Toric topology. 2012. arXiv:1210.2368.

[Frö90] R. Fröberg. On Stanley-Reisner rings. In Topics in algebra, volume 26, pages 57-70. Banach Center Publ., 1990.

[Gol62] E. S. Golod. On the homology of some local rings. In Soviet Math. Dokl, volume 3, pages 745-748, 1962.

[GS] D. R. Grayson and M. E. Stillman. Macaulay2, a software system for research in algebraic geometry. Available at http://www.math.uiuc.edu/Macaulay2/.

[HH13] J. Herzog and C. Huneke. Ordinary and symbolic powers are Golod. Advances in Mathematics, 246:89-99, 2013. 
[Hoc77] M. Hochster. Cohen-Macaulay rings, combinatorics, and simplicial complexes. In Ring theory II (Proc. Second Oklahoma Conference), pages 171-223. Marcel Dekker, Inc., 1977.

[HRW99] J. Herzog, V. Reiner, and V. Welker. Componentwise linear ideals and golod rings. Michigan Math. J, 46(2):211-223, 1999.

[IK14] K. Iriye and D. Kishimoto. The fat wedge filtration and a homotopy decomposition of a polyhedral product. arXiv:1412.4866v3, 2014.

[Jöl06] M. Jöllenbeck. On the multigraded Hilbert and Poincaré-Betti series and the Golod property of monomial rings. Journal of Pure and Applied Algebra, 207(2):261-298, 2006.

[LPWZ09] D.-M. Lu, J. H. Palmieri, Q.-S. Wu, and J. J. Zhang. $A_{\infty}$ structure on Ext-algebras. Journal of Pure and Applied Algebra, 213(11):2017-2037, 2009.

[Val14] B. Valette. Algebra + Homotopy = Operad. In Symplectic, Poisson, and Noncommutative Geometry. Cambridge University Press, 2014.

Universität Osnabrück, FB Mathematik/Informatik, 49069 Osnabrück, GerMANY

E-mail address: lukas.katthaen@uos.de 